\title{
Environmental Metrics to Drive a Cultural Change: Our Green Eco-Label
}

\author{
Ulrich Onken, Agnieszka Koettgen, Holger Scheidat, Patrick Schueepp, and Fabrice Gallou*
}

\begin{abstract}
A novel Green Chemistry Process Scorecard was developed to assess the environmental impact of chemical production processes to manufacture the Active Pharmaceutical Ingredients (API) within our portfolio. These new metrics not only cover the resource consumption from the overall chemical synthesis, but also consider the use of Substances of Concern and the number of chemical transformations. The Process Mass Intensity (PMI), i.e. the ratio of accumulated kilogram quantities of materials per kilogram of API, is used to quantify the resource consumption. An 'eco-label' for specific APIs is used to visualize the environmental impact from their chemical synthesis. For an overview of the environmental impact of a complete product portfolio, a diagram of $\mathrm{PMI}$ or total waste quantity vs. the number of synthetic steps can also be used as a visualization tool to identify chemical syntheses with a high need for process improvements. Implementation of this process led to a dramatic change of mindset within the organization. It now supports and drives the decision making at Chemical and Analytical Development, and helps to trigger new projects more readily for sustainability reasons.
\end{abstract}

Keywords: Green chemistry · Metrics · Sustainability

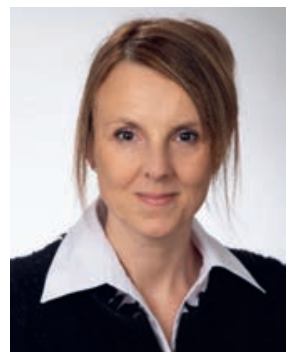

Agnieszka Köttgen is an organic chemist with a $\mathrm{PhD}$ from the University of Zurich (2006) and a Postdoc from the University of Basel. She has worked in different companies in the areas of polymer synthesis, analytics, and chemical outsourcing. She joined Novartis in 2013, and is now working as a process chemist managing production campaigns for APIs in the development phase.

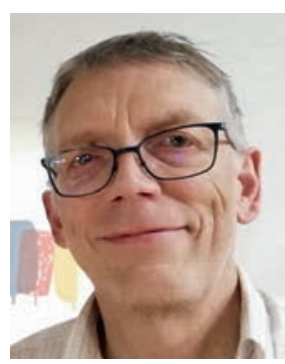

Ulrich Onken received his $\mathrm{PhD}$ from the University of Münster (Germany) in the field of heterogeneous catalysis (1987). After postdoctoral research at the University of Notre Dame (USA), he joined Ciba-Geigy in Basel (Switzerland) in 1989. Working in Chemical Development at Ciba-Geigy and later on Novartis he has developed and scaled-up several chemical manufacturing processes for APIs, with focus on hazardous reagents and high throughput. Throughout his career, Ulrich has driven green chemistry and sustainability efforts.

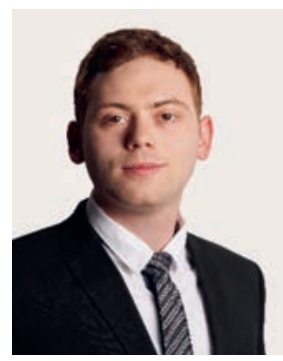

Patrick Schueepp just completed his MSc from the University of Zurich (2019) in the field of business and sustainable chemistry. Through his early academic curriculum, he already had strong industrial experience with internship at Roche (2009-15) and Novartis (2018-19) where he was exposed to and learnt chemical and analytical techniques, and developed broad awareness of the pharmaceutical industry.

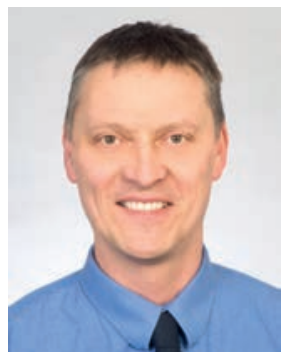

Holger Scheidat received his $\mathrm{PhD}$ from the University Erlangen-Nürnberg (1996) in the field of chemical vapor deposition. He joined Chemical Development at Novartis in 1996 as chemical engineer, and held various roles of growing responsibility within the organization. He is currently manufacturing officer and responsible for the HSE and GMP compliant production of APIs used for clinical trials.

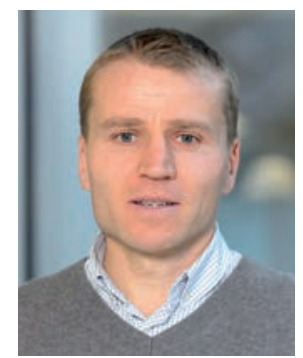

Fabrice Gallou received his $\mathrm{PhD}$ from The Ohio State University (2001) in the field of natural products total synthesis. He then joined Chemical Development at Boehringer Ingelheim, USA, working as a process chemist responsible for route scouting and supply of early phase programs. He subsequently moved in 2006 to Chemical Development at Novartis, Switzerland, as a process development chemist, and became in 2008 responsible for global scientific activities, overseeing development and implementation of practical and economical chemical processes for large-scale production of APIs. His research interests lie in the research and development of sustainable synthetic methodologies intended for large scale implementation.

\section{Introduction}

Developing a sustainable chemical synthesis is one of the ultimate missions of chemical development. ${ }^{[1]}$ About a decade ago, the Chemical and Analytical Development Green Chemistry Focus Group introduced the well-accepted Process Mass Intensity (PMI), as an index for measuring the greenness of a process. ${ }^{[2]}$ The PMI is intended to understand how much mass of material (in- 
cluding starting materials, reagents, solvents and water) is needed to manufacture one kilogram of a product. The quantitative assessment can be used to establish a benchmark, to identify areas for improvement and to measure achievements made in process development. While the implementation of this first set of metrics helped us significantly raise awareness on the criticality of our environmental footprint, some of us felt a limited sense of urgency and criticality within the organization. This brought us to the idea of designing our own concept, to not only address the technical needs via a quantitative aspect, but also affect the mindset and contribute to a change of culture in a qualitative manner. In an attempt to further strengthen our social responsibility in the environmental area, we indeed envisioned to expand on this concept and establish a more readily understandable and impactful process. The new business process, the Green Chemistry Process Scorecard, ultimately led to a standardized approach and a portfolio overview of the environmental impact of all our processes (see Fig. 1).

Our goal was to design an environmental sustainability label to rapidly visualize environmental impact ('greenness' of the process). The tool and business process is intended to be easily understandable for every associate within our large organization, from scientists of various backgrounds, to managers, colleagues in finance or legal... and can even be used for outside communication. It is user-friendly, allowing project teams to rapidly recognize areas for improvement.

\section{Raising Awareness and Engagement via Designing our own Eco-label}

At the onset of our efforts, we were conscious of the importance to get the buy-in from the whole organization. It was critical to engage our co-workers in this cultural journey to have a chance to make a significant and sustainable impact. Our strategy to address this point was to include early in our efforts diverse populations (various generations, e.g. baby-boomers, Gen $\mathrm{X}, \mathrm{Y}$, millennials, various areas of expertise ...) without boundaries across the technical expertises. The temptation would indeed have been to make it a restrictive technical exercise that we would subsequently struggle to communicate clearly and efficiently. Our first practical inclusive exercise consisted in engaging the organization in creating our own design for the green label. Inspired by the standard A/B/C/D/E eco-scale utilized for cars or electrical appliances for example, we aimed at measur- ing the sustainability of our processes with a Novartis' specific image that would promote pride and engagement in our midst. We constituted a group of a dozen professionals taken from various line functions, both technical (research, chemical development, production, biological, toxicological), and non-technical (project manager, human resources expert, communication experts). Through this process, we collected feedback and various thoughts that opened our minds and seeded novel ideas. From this first round of brainstorming, the need for a common currency appeared critical. While we would have simply utilized the standard scientific jargon, it became clear that we should utilize a currency that would speak to anyone for optimal accessibility. The current public pressure had allowed such opportunity to implement the carbon dioxide $\left(\mathrm{CO}_{2}\right)$ release as such common currency and we decided to follow this trend. Indeed, this is now a well-accepted scale on daily basis, and an important criteria for selection for cars, fridges, ... (see Fig. 2)

\subsection{Carbon Dioxide Equivalent and $\mathrm{CO}_{2}$ Release}

The various contributions can be translated into the release of carbon dioxide as a common currency to best communicate with all external stakeholders. Thus the quantity of carbon dioxide released to atmosphere per kilogram of API produced can be used as a universal unit of measurement for the ecological footprint. All contributors to the environmental footprint were assessed and clustered into four main categories:

From an analysis of several chemical manufacturing processes developed internally, the main contributor (>90\%) to the carbon dioxide release is clearly the incineration of organic waste; organic solvents accounting for about two thirds of mass utilization.

Another significant contribution is the wastewater treatment which in the worst case is done by incineration.

Energy consumption by manufacturing operations contributes to no more than $1 \%$ of the carbon dioxide release. This came after careful evaluation of several benchmark processes and is regularly revisited to make sure no critical input is omitted. It nevertheless seems that standard chemical processes do not consume meaningful amounts of energy compared to the various other footprints. For the sake of simplicity, this is therefore currently omitted in most processes.

Transport, operation of building facilities, plant cleaning, packaging and any lab process development activities were not

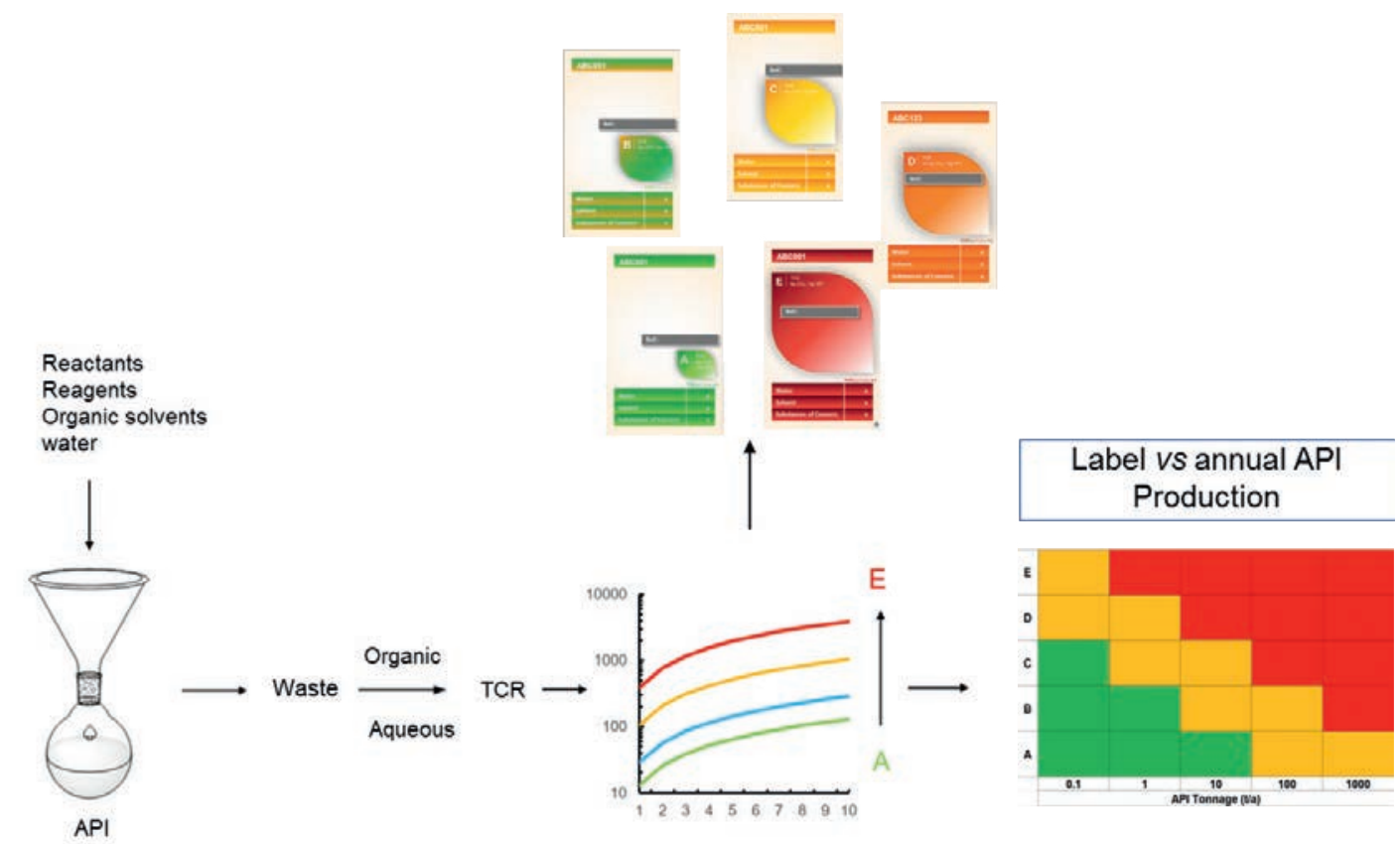

Fig. 1. Overview of our overall concept and visualization. 


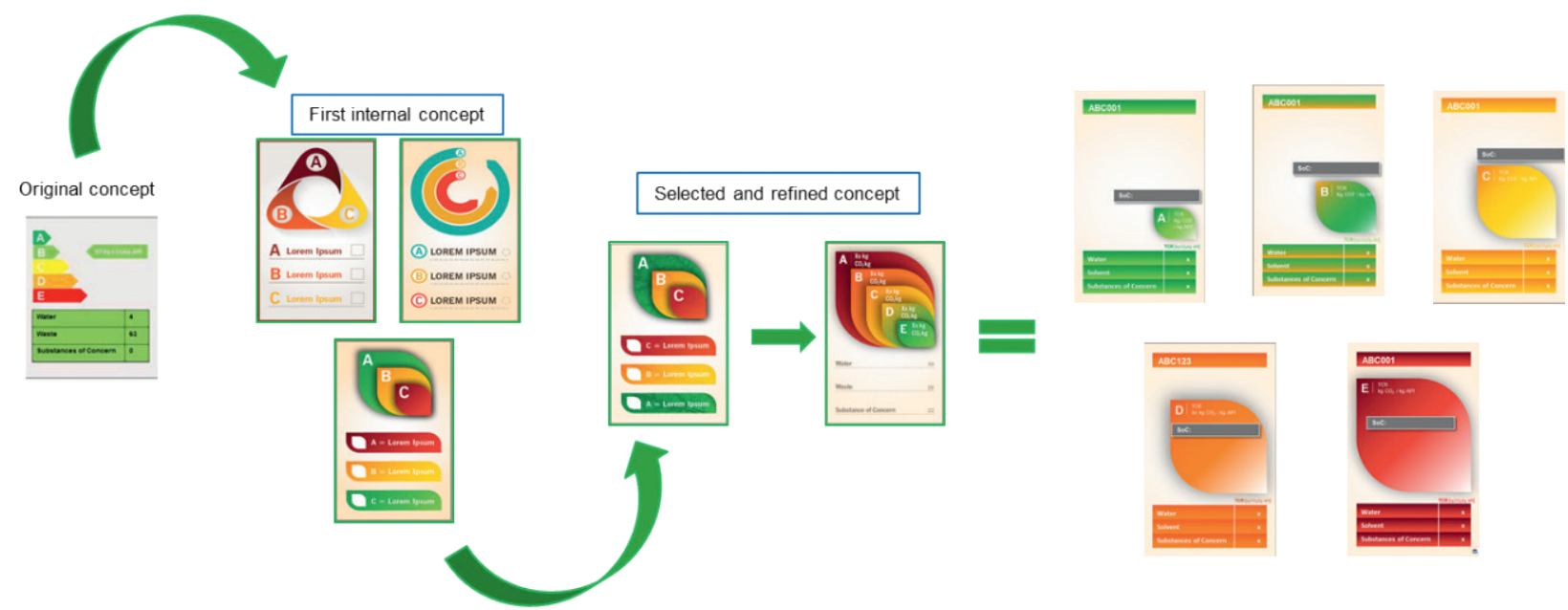

Fig. 2. Evolution of our visualization concept.

considered in this assessment as considered too specific to the plants and their mode of operation (dedicated plant, or multi-purpose plant with more or less fast turn-over required).

The real cultural breakthrough that came from our inclusive approach was the power of engaging and aligning the organization when working on a topic of intrinsic interest. This was indeed the case here, not just with the scientific and technical peers whose daily business essentially rely on implementing best sustainable practices, but also for the non-technical colleagues. The latter came as very passionate on the topic and great contributors, although not necessarily impacted by the outcome of the exercise. The passion and further drive such focus could generate were obvious bonuses that we did not want to miss. We therefore started elaborating and proposing options for our green label until a couple of specific advanced options were identified. At this stage, we asked our global chemical development organization to select via a vote the label we would be implementing. It is of course not quantitatively demonstrated how much we gained in this inclusive process, but more than four years after its implementation, we can see that the penetration and adherence to the business process are effective, despite minimum governance and no hierarchical push.

\section{Dimensions of a Process Scorecard}

Each chemical manufacturing process has an ecological impact on the environment, due to the consumption of raw materials including reagents, solvents and water, the disposal of process waste, the abatement of wastewater and due to energy consumption (e.g. for pumps and heating/cooling operations).

As basic guidelines, and based on the twelve principles of green chemistry, ${ }^{[1 d]}$ factors that improve the greenness of the chemical synthesis for an API should be taken into consideration: the number of chemical transformations should be minimized, functionalities that do not contribute to the API (e.g. protecting groups, chiral auxiliaries) should be minimized and catalysis considered instead, molar yield should be maximized, mass should be utilized optimally: solvent and water amounts should be minimized, and further recycled and/or reused, excess of reagents and catalyst loading should be minimized, and solvent and reagents should be carefully selected utilizing, for example, the great methodologies that the chemical community has came up within the past few years.

\subsection{Raw Materials}

Many of the raw materials used for the manufacture of an API have been produced by organic synthesis. Therefore each of them already carries with it its own contribution to the ecological footprint of the API synthesis. While under the term 'Life Cycle
Analysis' estimates exist for the environmental impact of some common raw materials, it is unknown for most purchased raw materials. Sufficient information to estimate their impact is hard or impossible to obtain from the suppliers. We are currently working on a way to accurately assess their footprint, and this impact is yet not taken into account, striving to go back as far as possible within synthesis to measure the largest possible fraction of the overall chemical footprint.

\subsection{Organic Waste}

If not converted into the desired product, raw materials end up as organic process waste which is typically dissolved in organic solvents. If no recovery and recycling is performed, the waste has to be incinerated. The carbon dioxide release from incineration for typical organic compounds is between 1.5 and $3 \mathrm{~kg}$ carbon dioxide per $\mathrm{kg}$ waste. The carbon dioxide release from incineration of a representative solvent mixture such as a THF/methanol/heptane mixture results in $2.3 \mathrm{~kg}$ carbon dioxide per $\mathrm{kg}$. This figure is now being used as an approximation for the carbon dioxide release for typical organic solvent waste.

\subsection{Wastewater}

Whereas process water is abundant and its use does not cause severe harm to the environment, it will end up as process wastewater. The wastewater contains salts and/or organic components which require treatment, in order to avoid causing harm to the environment.

If wastewater contains mainly biodegradable components and limited salt content, it can usually be released to an Effluent Treatment Plant (ETP) which is available at most chemical manufacturing sites. After biological treatment, which releases some carbon dioxide, it is usually safe to release the purified wastewater into streams or into the sea. If the wastewater quality is not adequate for release to an ETP, it has to be disposed of by other means which can involve special techniques such as wet-air oxidation or incineration. Often wastewater incineration requires the addition of extra fuel which may be waste solvent or heavy oil $(150 \mathrm{~kg}$ of fuel per metric ton of wastewater). The typical carbon dioxide release from wastewater incineration was estimated to $0.628 \mathrm{~kg}$ carbon dioxide $/ \mathrm{kg}$ wastewater.

In the current version of the Scorecard, there is no distinction made between the wastewater released to ETP or wastewater incinerated. The calculations are based on the assumption that the process wastewater is incinerated as a worst-case scenario. Further changes to this evaluation process are being implemented to introduce greater granularity.

Overall, this led us to the following definition of the total carbon dioxide release (TCR): 
Total $\mathrm{CO}_{2}$ Release $(\mathrm{TCR})=($ PMI organic $\times 2.3+$ PMI water x 0.63 ) per $\mathrm{kg}$ API

With Process Mass Intensity $=\underline{\text { Quantity of raw materials input }(\mathrm{kg})}$ Quantity of bulk API out $(\mathrm{kg})$

\section{Rating of Carbon Dioxide Release Data}

Various options were considered to rate the carbon dioxide release. For example, kilogram waste per kilogram of API produced, or kilogram waste per patient and therapy, or kilogram waste taken as an absolute figure, e.g. to obtain the environmental impact for a manufacturing site.

The kilogram waste per kilogram of API produced ultimately translated into carbon dioxide release was eventually selected as a more meaningful and instructive unit.

\subsection{Simplified Approach for Carbon Dioxide Contributions to the Scorecard}

Contributions to the environmental impact from raw materials and waste are complex. The following simplifications were therefore introduced to enable a simple and robust workflow to generate a process scorecard:

- The carbon dioxide release from a chemical synthesis is used as the key figure to quantify the ecological impact from raw materials, the disposal of waste and the disposal of wastewater.

- When looking at the kg-based figure of the PMI alone, the contributions from wastewater may be over-estimated (organic waste has a superior footprint compared to water for example). Therefore the PMI figure is also converted into $\mathrm{kg}$ carbon dioxide released per $\mathrm{kg}$ API, differentiating the various contributions. The calculated TCR is used to determine the Scorecard rating.

- As previously mentioned, energy consumption for processing operations is currently neglected because of its small contribution to the total carbon dioxide release.

- The scope of the scorecard includes all chemical steps to produce the API for which Novartis owns the specific chemical process know-how.

- Plant cleaning operations are out of scope. They highly depend on the equipment, campaign duration, scale, and paradigm use of the specific plant.

- For simplicity reasons, all other activities not directly related to the chemical synthesis (transport, operation of building facilities, packaging and any lab process development activities) are considered out of scope in the first generation of our tool.

- Process wastewater is assumed to be incinerated as a worstcase scenario, i.e. there is no credit given if it can be released via an ETP. This results in a very conservative approach as a basic guidance for our tool (we want to capture opportunities and be as critical as possible).

\subsection{Substances of Concern}

Substances with major health concerns (mutagenic, teratogenic, reprotoxic or displaying other toxic properties) or with other major concerns for the environment (e.g. highly volatile solvents) ${ }^{[4]}$ are undesired in a chemical synthesis. The use or formation of such substances of concern should be avoided, e.g. by replacing toxic reagents with less toxic ones. ${ }^{[5]}$

There is no simple approach to identify such substances of concern or to assign ratings to substances according to the impact to the environment. For the Green Chemistry Scorecard, a simple qualitative approach was adopted to systematically report any undesirable chemical entity used, independent of its role and quantity (catalyst, reagent, solvent, reactant) The rationale was to flag systematically the use of undesirable chemicals, thus edu- cating the more junior associates especially, and simply trigger further questions.

The current process is the following:

- Compounds which are either listed in the candidate list of substances of very high concern (SVHC) at the European Chemicals Agency (ECHA), or flagged by the US Environmental Protection Agency (EPA)...,[3] or which have been labeled as problematic for use through our internal experience are considered as Substances of Concern. They are captured into our scorecard template and also includes their Chemical Abstracts registry numbers. The severity and extent of the list can be adapted based on organization culture and overall guidance.

- Each substance of concern present in a chemical synthesis is highlighted on the eco-label purely visually as a black label. The scorecard rating itself is not influenced by the substances of concern.

\subsection{Number of Synthesis Steps and Chemical Transformations}

The chemical complexity of a compound obviously has a large impact on resource consumption during its manufacture by chemical synthesis. Thus it is expected that the PMI for the synthesis increases with the number of processing steps. This is actually the case, however with large variations (Fig. 3). Benchmarks of hundreds of syntheses collected from the literature have allowed the generation of a good understanding of the standard correlation between the number of steps and the PMI. This is of course subject to specificity but for the most part, as demonstrated since then by other pharmaceutical leaders in the field, such a hypothesis proves valid and supports the decision-making processes in most cases.

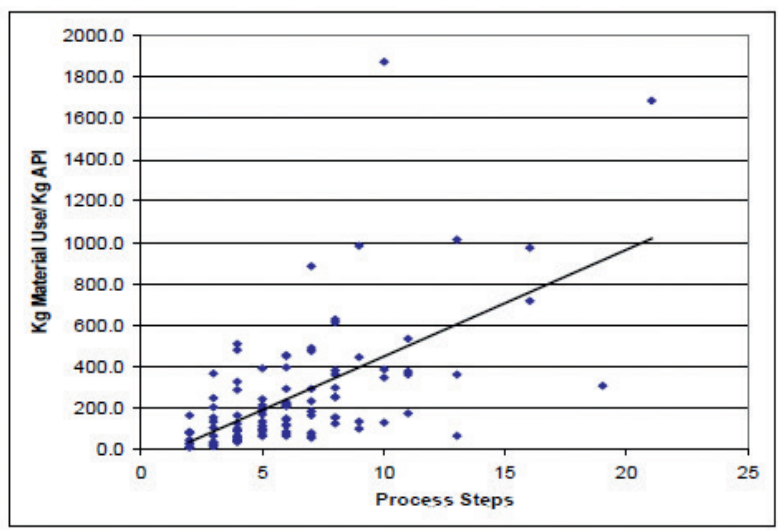

Fig. 3. Process Mass Intensity vs. number of process steps in chemical synthesis for a portfolio (assessment made in 2011).

\section{The Scorecard Template and the Green Eco-Label}

The current template to be used for the calculation of scorecard ratings is shown for reference. It is based on a PMI analysis. Another simple template can be found from the ACS Green Chemistry Pharmaceutical Industry Roundtable.

The cumulative PMI (all materials used) is calculated for all processes. In order to get the carbon footprint for the process (i.e. the total carbon dioxide release, TCR), the PMI for organic compounds (leading to organic waste) and the PMI for water (coming from wastewater incineration) are calculated separately. The PMIs are multiplied by 2.3 (2.3 kg carbon dioxide / $\mathrm{kg}$ organic waste) and 0.628 ( $0.628 \mathrm{~kg}$ carbon dioxide / $\mathrm{kg}$ waste water), respectively, and the sum is the total carbon dioxide release. This number is used to determine the Scorecard rating. This latter scoring has been constructed into a simple Excel spreadsheet to automatically provide the outcome (Fig. 4). 


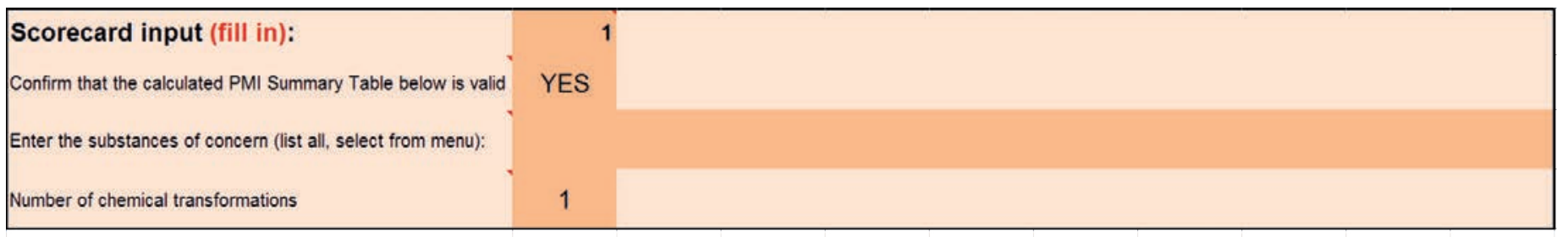

\begin{tabular}{|lcc|}
\hline Scorecard results & & \\
PMI / total CO2 release & $142 \mathrm{~kg} / \mathrm{kg} \mathrm{API}$ & $218 \mathrm{~kg} \mathrm{CO} 2 / \mathrm{kg} \mathrm{API}$ \\
Waste dimension & $77 \mathrm{~kg} / \mathrm{kg} \mathrm{API}$ & $177 \mathrm{~kg} \mathrm{CO} 2 / \mathrm{kg} \mathrm{API}$ \\
Water dimension & $65 \mathrm{~kg} / \mathrm{kg} \mathrm{API}$ & $41 \mathrm{~kg} \mathrm{CO} 2 / \mathrm{kg} \mathrm{API}$ \\
Substances of concern & 0 & 0 substance $(\mathrm{s})$ \\
Number of steps assessed & 2 & \\
Number of chemical transformations & 1 & \\
\hline
\end{tabular}

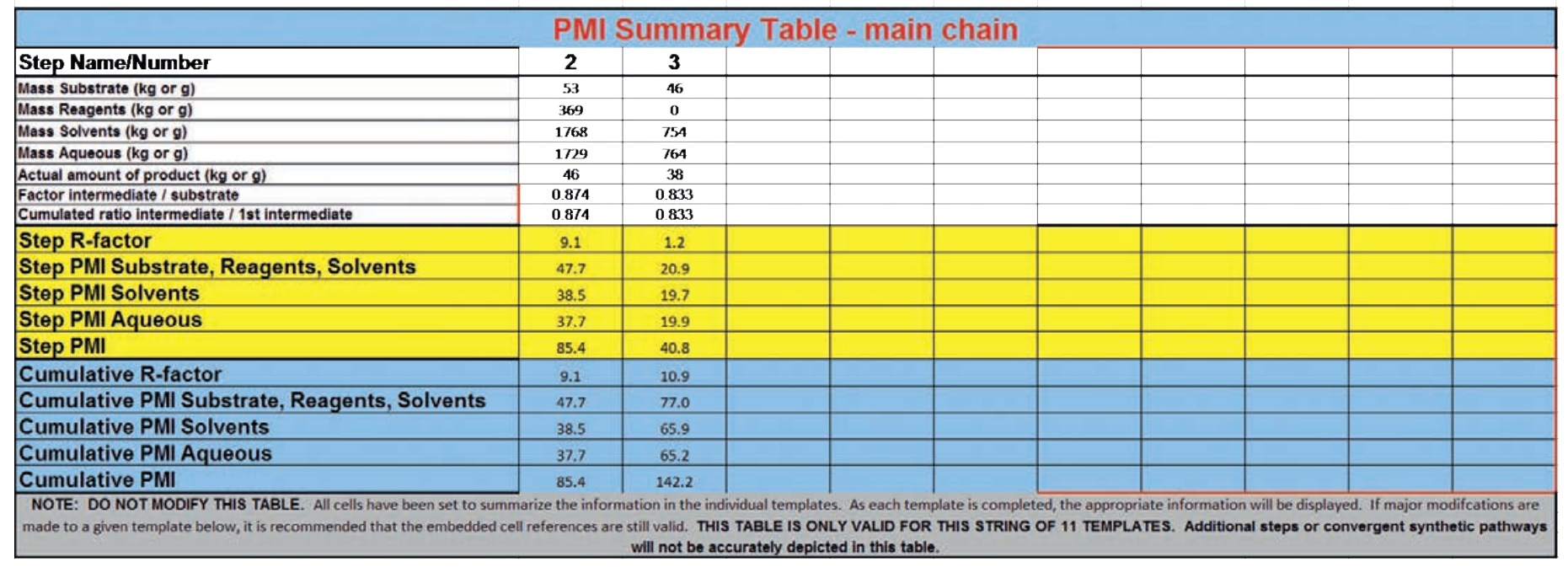

Fig. 4. Example for a Process Mass Intensity calculation scheme as used for the generation of a process scorecard.

In order to obtain the PMI and carbon dioxide figures in the scorecard, the outcome generated above is put in perspective of the number of chemical transformations.

For this, based on an analysis of our chemical portfolio over about a decade, specific scorecard ratings were assigned according to the TCR for the synthesis and the required number of transformations. Green labels ratings range from A (excellent footprint, low environmental impact and burden), which is the most favorable eco-label score, to E (poor footprint, high environmental impact and burden) (see Fig. 5).

\begin{tabular}{|c|c|c|c|c|}
\hline Transformations & TCR-AB & TCR-BC & TCR-CD & TCR-DE \\
\hline 1 & 13 & 28.6 & 104 & 390 \\
\hline 2 & 26 & 57.2 & 208 & 780 \\
\hline 3 & 39 & 85.8 & 312 & 1170 \\
\hline 4 & 52 & 114.4 & 416 & 1560 \\
\hline 5 & 65 & 143 & 520 & 1950 \\
\hline 6 & 78 & 171.6 & 624 & 2340 \\
\hline 7 & 91 & 200.2 & 728 & 2730 \\
\hline 8 & 104 & 228.8 & 832 & 3120 \\
\hline 9 & 117 & 257.4 & 936 & 3510 \\
\hline 10 & 130 & 286 & 1040 & 3900 \\
\hline 11 & 143 & 314.6 & 1144 & 4290 \\
\hline 12 & 156 & 343.2 & 1248 & 4680 \\
\hline
\end{tabular}

For example, for a seven-transformation synthesis, a TCR below 91 will give an eco-label of A, while a TCR between 200 and 728 will give a label $\mathrm{C}$. The boundaries were defined $a$ priori and refined within a couple of years in the pilot of our scorecard to discriminate sufficiently well our portfolio, and to result systematically in a manageable number of projects where activities should be triggered based on sustainability aspects.

The data for a complete portfolio can be visualized in a semilogarithmic plot of total carbon dioxide release and the number of transformations.

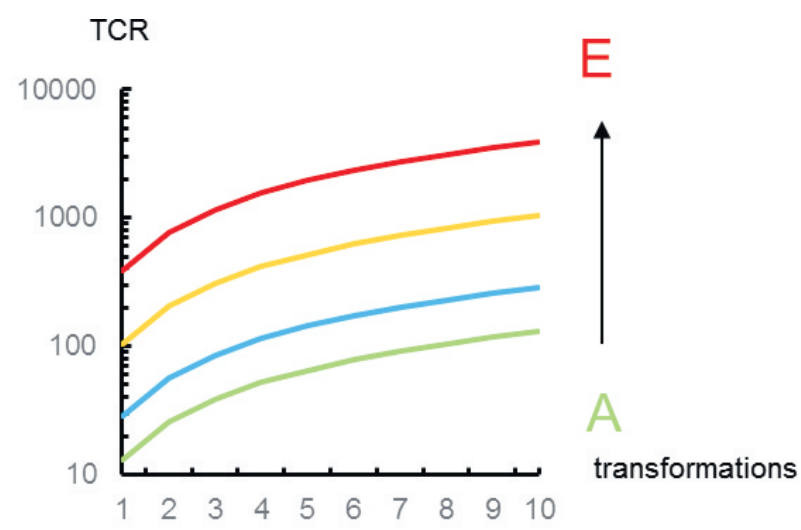

Fig. 5. Eco-label ratings: Boundaries between A, B, C, D, E ratings depending on the number of transformations and TCR, and Total Carbon Release as a function of the number of Chemical Transformations. 


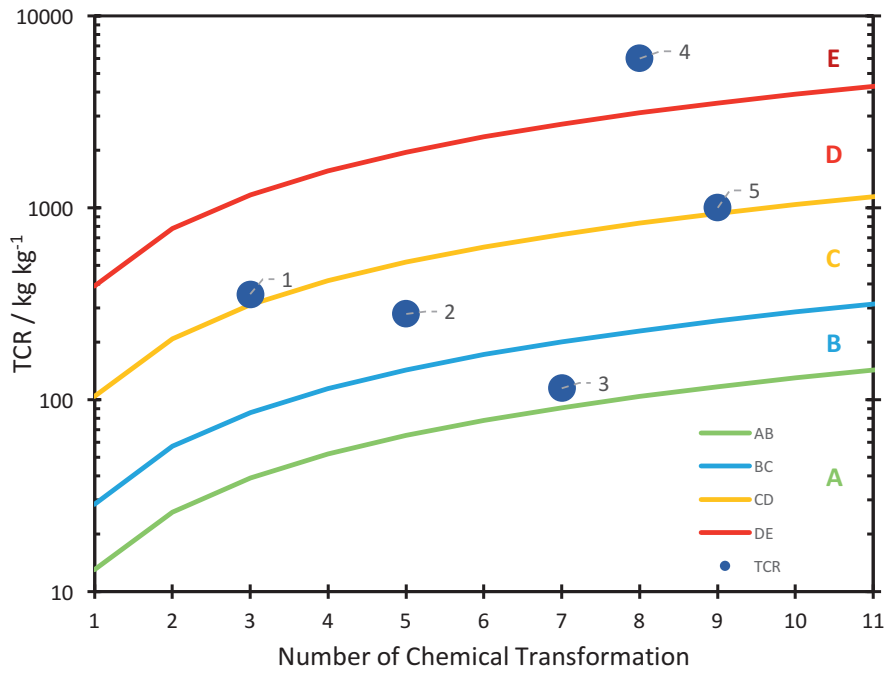

Fig. 6. Total $\mathrm{CO}_{2}$ Release data vs. the number of chemical transformations for the CHAD portfolio in 2016.

The data can also be represented in annual $\mathrm{kg}$ quantities of waste (for the predicted peak sales volume per project) in order to visualize the actual impact on the environment. Such diagrams may support decisions to set priorities for chemical process development and improvement on those projects where the expected improvement in terms of absolute waste reduction is high (Fig. 6).

\subsection{Eco-label and Visualization for a Product Portfolio}

In order to support decisions and trigger activities, we analyze our portfolio by calibrating it to the absolute amount of API required at peak volume. Such an approach allows us to focus on real impactful projects, in an absolute manner, and not necessarily get carried away on very low volume projects where there is little to gain ultimately. Fig. 7 indicates the expected annual waste volume from API manufacture at peak sales. This visualization strongly supports decisions on priorities for process improvement work since it shows where the highest potentials for the reduction of waste quantities are. A standard situation is exemplified below and our thought process is the following:

- For the low volume project 1, although the label is rather mediocre (D), one can see that the overall impact once put in perspective of the demand of the project at estimated peak sale is very low and somewhat inconsequential.

- In contrast, project 2, that has a slightly better green eco label (C), has more impact from an environmental aspect; the same holds true for project 3 , despite the even better green eco-label
(B). In such situations, our priorities would therefore go to project 3 first, then to 2, and finally 1 .

- A similar type of analysis can be made to rationalize the priorities between projects 4 and 5 . As a rule of thumb, we endeavor to do all critical high volume projects as even minute improvement can result in significant benefits in the final environmental footprint.

\subsection{Business Process}

The business process to generate, evaluate, and report the scorecard has been designed to educate as many associates as possible through the process, while maximizing synergies and opportunities for exchange. We have favored cross-functional exchanges, linking laboratory and pilot chemists to generate together the outcome. These chemists define the synthesis and manufacturing processes for the synthetic intermediates, including materials used and the mass balances. For each campaign, the plant chemist calculates the PMI and the total carbon dioxide release using the PMI tool which are reported in a Campaign Experience Report.

Based on this information, a departmental Green Chemistry Champion uses the scorecard methodology to assign an eco-label (A, B, C, D or E) with total carbon dioxide release and Substances of Concern as supplementary information. This process is also applied for projects coming from early development activities. To share the results with the project team, the Green Chemistry Champion reports them to the project leader, who communicates results back to his/her team. A central Green Chemistry Champion was seen as critical to ensure consistency, and anchor the organization behind such an important process.

The scorecard label with total carbon dioxide release and the number of chemical transformations is also used to conduct a periodic portfolio analysis by the Green Chemistry Champion. The result of the analysis is visualized as reported in Fig. 6 and, in alignment with scientific experts, recommendations to management are made to trigger activities in projects with poor and highly impactful environmental performance.

\section{Outlook}

The reported methodology in its first generation has now been going live for several years and drastically changed the mindset, providing a frame and a purpose behind our efforts in the field. It was important to us to start rapidly even with a less than perfect methodology, even just to raise awareness, the sense of urgency and responsibility within our population. The overall process being amenable to further improvement without significant changes is going through constant challenge and continuous improvement that we will report in due time.

Received: July 23, 2019

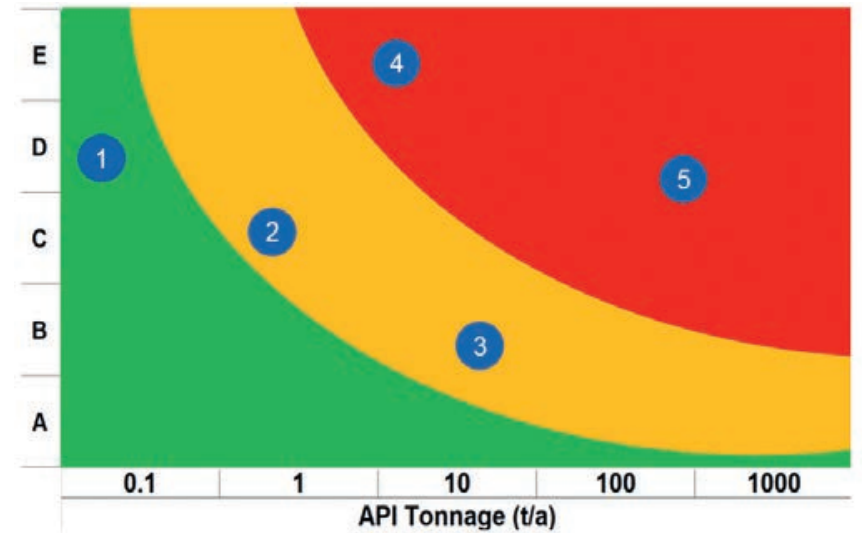

Fig. 7. Eco-labels as a function of expected annual tonnage at peak sales.
[1] a) R. A. Sheldon, ACS Sustain. Chem. Eng. 2018, 6, 32, doi:10.1021/ acssuschemeng.7b03505; b) P. T. Anastas, J. C. Warner, 'Green Chemistry: Theory and Practice', Oxford Univ. Press, New York, 1998, doi:10.1103/ PhysRevB.71.144303; c) ACS, 'Twelve Principles of Green Chemistry', 2018, https://www.acs.org/content/acs/en/greenchemistry/principles/12principles-of-green-chemistry.html, accessed: July 12, 2019; d) R. A Sheldon, Chemtech 1994, OSTI ID: 7109196, doi:10.2144/000113917; e) R. A. Sheldon, Green Chem. 2017, 19, 18, doi:10.1039/c6gc02157c; f) J. Andraos, M. L. Mastronardi, L. B. Hoch, A. Hent, ACS Sustain. Chem. Eng. 2016, 4, 1934, doi:10.1021/acssuschemeng.5b01555; g) F. Roschangar, J. Colberg, P. J. Dunn, F. Gallou, J. D. Hayler, S. G. Koenig, M. E. Kopach, D. K. Leahy, I. Mergelsberg, J. L. Tucker, R. A. Sheldon, C. H. Senanayake, Green Chem. 2017, 19, 281, doi:10.1039/c6gc02901a; h) F. Roschangar, R. A. Sheldon, C. H. Senanayake, Green Chem. 2015, 17, 752, doi:10.1039/ c4gc01563k; i) F. Roschangar, Y. Zhou, D. J. C. Constable, J. Colberg, D. P. Dickson, P. J. Dunn, M. D. Eastgate, F. Gallou, J. D. Hayler, S. G. Koenig, M E. Kopach, D. K. Leahy, I. Mergelsberg, U. Scholz, A. G. Smith, M. Henry, J. Mulder, J. Brandenburg, J. R. Dehli, D. R. Fandrick, K. R. Fandrick, F. Gnad-Badouin, G. Zerban, K. Groll, P. T. Anastas, R. A. Sheldon, C. H. 
Senanayake, Green Chem. 2018, 20, 2206, doi:10.1039/c8gc00616d; j) M. G. T. C. Ribeiro, D. A. Costa, A. A. S. C. Machado, Green Chem. Lett. Rev. 2010, 3, 149, doi:10.1080/1751825100362337; k) M. G. T. C. Ribeiro, S. F. Yunes, A. A. S. C. Machado, J. Chem. Educ. 2014, 91, 1901, doi:10.1021/ ed400421b; 1) K. Van Aken, L. Strekowski, L. Patiny, Beilstein J. Org. Chem. 2006, 2, 3, doi:10.1186/1860-5397-2-3; m) D. Kaiser, J. Yang, G. Wuitschik, Org. Process Res. Dev. 2018, 22, 1222, doi:10.1021/acs.oprd.8b00199; n) D. K. Leahy, E. M. Simmons, V. Hung, J. T. Sweeney, W. F. Fleming, M. Miller, Green Chem. 2017, 19, 5163, doi:10.1039/c7gc02190a; o) R. Dach, J. J. Song, F. Roschangar, W. Samstag, C. H. Senanayake, Org. Process Res Dev. 2012, 16, 1697, doi:10.1021/op300144g; p) A. D. Curzons, C. JiménezGonzález, A. L. Duncan, D. J. C. Constable, V. L. Cunningham, Int. J. Life Cycle Assess. 2007, 12, 272, doi:10.1065/lca2007.03.315; q) C. JiménezGonzález, A. D. Curzons, D. J. C. Constable, V. L. Cunningham, Int. J. LCA 2004, 9, 114, doi:10.1007/BF02978570; r) P. Saling, A. Kicherer, B. Dittrich-Krämer, W. Z. Wittlinger, I. Schmidt, W. Schrott, S. Schmidt, Int. J. LCA. 2002, 7, 203, doi:10.1007/BF02978875; s) P. Saling, R. Maisch, M. Silvani, N. König, Int. J. LCA 2005, 10, 364, doi:10.1065/lca2005.08.220; t) A. Kicherer, S. Schaltegger, H. Tschochohei, B. F. Pozo, Int. J. LCA 2007, 12, 537, doi:10.1065/lca2007.01.305; u) T. V. T. Phan, C. Gallardo, J. Mane, Green Chem. 2015, 17, 2846, doi:10.1039/c4gc02169j; v) Green Motion ${ }^{\mathrm{TM}}$ by MANE. Available at: https://www.mane.com/innovation/green-motion, accessed: July, 12, 2019; w) L. Leseurre, C. Merea, S. Duprat de Paule, A Pinchart, Green Chem. 2014, 16, 1139, doi:10.1039/c3gc42201a.

[2] C. Jimenez-Gonzalez, C. S. Ponder, Q. B. Broxterman, J. B. Manley, Org. Process Res. Dev. 2011, 15, 912, doi:10.1021/op200097d.

[3] a) Candidate list of substances of very high concern (SVHC) at ECHA http://echa.europa.eu/candidate-list-table, accessed: July 12, 2019; b) Toxic
Substance Control Act (TSCA) at EPA, https://www.epa.gov/assessing-andmanaging-chemicals-under-tsca, accessed: July 12, 2019.

[4] a) D. Prat, O. Pardigon, H.-W. Flemming, S. Letestu, V. Ducandas, P. Isnard, E. Guntrum, T. Senac, S. Ruisseau, P. Cruciani, P. Hosek, Org. Process Res. Dev. 2013, 17, 1517, doi:10.1021/op4002565; b) D. Prat, A. Wells, J. Hayler, H. Sneddon, R. McElroy, S. Abou-Shehada, Green Chem. 2015, 18, 288, doi:10.1039/c5gc01008j; c) K. Alfonsi, J. Colberg, P. J. Dunn, T. Fevig, S. Jennings, T. A. Johnson, H. P. Kleine, C. Knight, A. Nagy, D. A. Perry, M. Stefaniak, Green Chem. 2008, 10, 31, doi:10.1039/b711717e; d) C. JiménezGonzalez, A. D. Curzons, D. J. C. Constable, V. L. Cunningham, Clean Technol. Environ. Policy 2005, 7, 42, doi:10.1007/s10098-004-0245-z; e) R. K. Henderson, C. Jimenez-Gonzalaz, D. C. Constable, S. R. Alston, G. A. Inglis, G. Fisher, J. Sherwood, S. P. Binks, A. D. Curzons, Green Chem. 2011, 13, 854, doi:10.1039/c0gc00918k; g) C. M. Alder, J. D. Hayler, R. K. Henderson, A. M. Redman, L. Shukla, L. E. Shuster, H. F. Sneddon, Green Chem. 2016, 18, 3879, doi: 10.1039/C6GC00611F; h) D. Prat, J. Hayler, A. Wells, Green Chem. 2014, 16, 4546, doi:10.1039/c4gc01149j; i) F. P. Byrne, S. Jin, G. Paggiola, T. H. M. Petchev, J. H. Clark, T. J. Farmer, A. J. Hunt, C. R. McElroy, J. Sherwood, Sustain. Chem. Process. 2016, 4, 7, DOI 10.1186/ s40508-016-0051-z.

[5] a) ACSGCIPR, 'Reagent guides', 2015, https://reagents.acsgciprorg/, accessed: July 22, 2019; b) J. P. Adams, C. M. Alder, I. Andrews, A. M. Bullino, M. Campbell-Crawford, M. G. Darcy, J. D. Hayler, R. K. Henderson, C. A. Oare, I. Pendrak, A. M. Redman, L. E. Shuster, H. F. Sneddon, M. D. Walker, Green Chem. 2013, 15, 1542, doi:10.1039/c3gc40225h; c) R. K. Henderson, A. P. Hill, A. M. Redman, H. F. Sneddon, Green Chem. 2015, 17, 945, DOI:10.1039/c4gc01481b. 